\title{
DSC Cure Studies of High Performance Epoxy Resins I. The Reaction with 4,4'-Diaminodiphenyl Sulfone (DDS)
}

\author{
Jyong Sup SHIM, Weon LeE, and Jyongsik JANG \\ Department of Chemical Technology, Seoul National University, \\ Sinlimdong Kwanakgu, Seoul 151-742, Korea \\ * Polymer Composites Laboratory, Korea Institute of Science \& Technology, \\ P.O. Box 131, Cheongrayang, Seoul 131, Korea
}

(Received September 14, 1990)

\begin{abstract}
Dynamic and isothermal cure kinetics of three different formulations composed of a new tetraglycidyl epoxy resin and diaminodiphenyl sulfone (DDS) were analyzed by differential scanning calorimetry. An autocatalytic kinetic model with the overall reaction order of 2 was modified for adequately describing the cure kinetics. Compared to previous calculation of kinetic parameter, the modified calculation fitted well with the experimental results. Higher cure temperature and higher DDS concentration led to an increase in the cure rate. Cure process of epoxy was dependent on the structure and functional group of epoxy resin. At the low DDS concentration, second exotherm peak was observed at approximately $50 \%$ conversion. Dynamic kinetic parameters were larger than isothermal kinetic parameters.

KEY WORDS Epoxy Resin / Diaminodiphenyl Sulfone Curing Agent / Cure Rate / Dynamic Thermogram / Isothermal Experiment / Degree of Conversion/Cure Time / Cure Temperature / Reciprocal Peak Temperature /
\end{abstract}

Epoxy resins have been widely used for many applications after late 1940's. ${ }^{1-5}$ Especially the epoxy resins composed of tetraglycidyl-4,4'diaminodiphenylmethane (TGDDM) and diaminodiphenyl sulfone (DDS) are currently used as principal matrix resins of high-performance composites in aircraft and aerospace industries. However, hot/wet performance of TGDDM/ DDS system has been considered unsuitable because of high levels of its moisture absorption.

Recently, new tetraglycidyl resins/curative systems, which have much improved hot/wet performance and high glass transition temperature over TGDDM/DDS system, have been developed by Shell Chemical Company. ${ }^{6-9}$ These resins are $N, N, N^{\prime}, N^{\prime}$-tetraglycidyl- $\alpha, \alpha^{\prime}$ bis(4-aminophenyl)-p-diisopropylbenzene (EPON HPT Resin 1071, abbreviated to TGAP) and $N, N, N^{\prime}, N^{\prime}$-tetraglycidyl- $\alpha, \alpha^{\prime}-$ bis(3,5-dimethyl-4-aminophenyl)-p-diisopropylbenzene (EPON HPT Resin 1072, abbreviated to TGMAP). A fundamental study of curing process in this resin is essential for the resin handling, prepreg fabrication, and the optimization of resin based composite processing.

In general, cure reactions of the amine-epoxy resin show complex kinetics by an initial acceleration due to autocatalytic effect. The later postgelation stages may exhibit retaradation as the mechanism becomes diffusion controlled. Kinetic expression describing the autocatalytic behaviour of epoxy cure has been discussed in the literature. ${ }^{10-12}$ These autocatalytic reaction models suggest that the cure process is governed by second reaction order.

Although several studies have been performed to evaluate the mechanical properties of cured TGAP resin for composite applica- 
tions, they give very limited information concerning cure kinetics. It is the purpose of this study, therefore, to analyze the cure kinetics of several TGAP Resins/DDS formulations by differential scanning calorimetry (DSC). In addition, the kinetic parameters measured by dynamic and isothermal scans have been compared with various epoxy resin formulations.

\section{EXPERIMENTAL}

\section{Materials}

The epoxy resin used in this study was EPON HPT 1071 supplied by Shell Chemical Company. This resin is technical grade of $N, N, N^{\prime}, N^{\prime}$-tetraglycidyl- $\alpha, \alpha^{\prime}$-bis(4-aminophenyl)-p-diisopropylbenzene (epoxy equivalent weight: $166 \mathrm{~g} \mathrm{~mol}^{-1}$ ). The curing agent was 4,4'-diaminodiphenyl sulfone (DDS), hardner HT 976, obtained from Ciba Geigy Ltd. The structures of EPON HPT 1071 and DDS curing agent are given in Figure 1. These materials were used as supplied without further purification. Three sorts of the epoxy compounds, HD1, HD2, and HD3, were prepared by changing the molar ratio of diamine (DDS)/ epoxide (TGAP), $\gamma$. The values of $\gamma$ of HD1, HD2, and HD3 were 1.0, 0.71, and 0.54, respectively. The curing agent in acetone was dissolved into the epoxy resin and the mixture was mixed completely with the mechanical stirrer, followed by evaporating the solvent in vacuum ovent at room temperature.

\section{Methods}

The thermal and kinetic data were obtained using Perkin-Elmer 4 differential scanning calorimeter coupled to a microprocess controller. The instrument was calibrated for temperature and enthalpy with high purity indium. Samples of $5-15 \mathrm{mg}$ in crimpped aluminium pans were cured in the DSC under dynamic and isothermal condition at nitrogen atmosphere. An empty pan was used as the reference material.

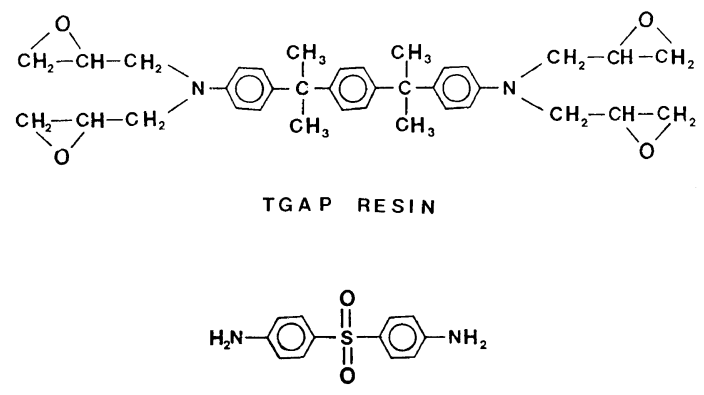

DIAMINODIPHENYL SULFONE(DDS)

Figure 1. The structure of TGAP resin and DDS curing agent.

Isothermal experiments were run for HD13 at fiber different temperatures. For isothermal cure, the DSC was first equilibrated at the present cure temperature and then the sample was introduced into the DSC cell. The thermal equilbrium of the sample and the reference holder was attained in less than $1 \mathrm{~min}$ after sample insertion.

The dynamic experiments were carried out at heating rate in the range of $2.5-20 \mathrm{~K} \mathrm{~min}^{-1}$. The baseline was obtained from a second scan on the fully cured resin.

The cure rate equation used in this study was

$$
\dot{\alpha}=\mathrm{d} \alpha / \mathrm{d} t=\left(k_{1}+k_{2} \alpha^{m}\right)(1-\alpha)^{n}
$$

where $\alpha$ is the extent of cure, $k_{1}$ and $k_{2}$ are the kinetic rate constants, and $m$ and $n$ are kinetic exponents. The reaction rate was determined directly from DSC thermograms

$$
\mathrm{d} \alpha / \mathrm{d} t=(\mathrm{d} H / \mathrm{d} t) / H_{\text {com. }} .
$$

where $H_{\text {com. }}$ is the complete heat of cure. In order to get the degree of conversion, the DSC curves were integrated and the partial areas as a function of time were normalized with respect to $H_{\text {com. }}$ and sample weight.

\section{RESULTS AND DISCUSSION}

\section{Dynamic Cure Kinetics}

DSC scans were run for the HD1-3 at five 
different heating rate. The heat of cure is obtained from curve displacement as a function of temperature. Values of the heat of cure $\left(H_{\mathrm{c}}\right)$ was independent of heating rate at a given DDS concentration. However, the heat of cure progressively decreases with increasing DDS concentration. At a scan speed of $10^{\circ} \mathrm{C} \mathrm{min}^{-1}$, the values of $H_{\mathrm{c}}$ are; $489.3 \mathrm{Jg}^{-1}$ for $\mathrm{HD} 1$ $556.5 \mathrm{Jg}^{-1}$ for HD2, and $598.1 \mathrm{Jg}^{-1}$ for HD3. This fact indicates that the extent of consumption of epoxy groups by other reactions than primary amine-epoxide is enhanced at the low concentration of amine. The reaction of epoxide of epoxide with amine involves the following overall reactions. ${ }^{1}$

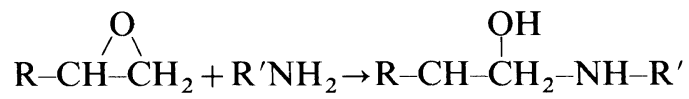

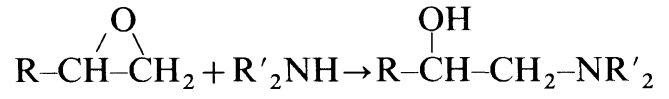

The hydroxyl groups formed by the amineepoxide addition are active catalyst. Furthermore, when the amine is present in less than stoichiometric concentration, reaction of epoxide and hydroxyl may occur.

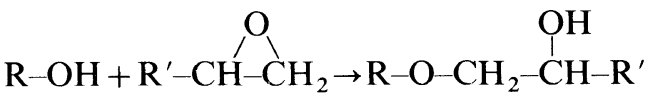

Typical modes of dynamic DSC thermogams are shown in Figure 2. Dynamic DSC scans were performed on samples of HD1, HD2 and HD3, whose diamine/epoxide ratios are 1.0, 0.71 , and 0.54 at heating rates of 2.5, 5.0, 10.0, and $20.0^{\circ} \mathrm{C} \mathrm{min}^{-1}$. From the figure, several noteworthy phenomena have been observed: at a constant curing agent, the initial cure temperature and the peak temperature increase with increasing the heating rate. The total heat of cure estimated from the area under the exotherm is independent of the heating rate. At a constant heating rate, the onset of curing

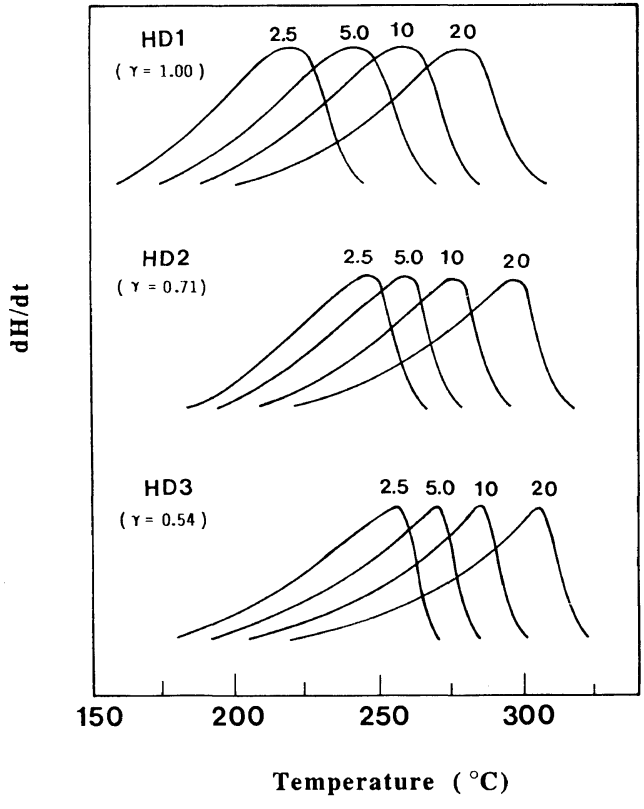

Figure 2. DSC dynamic thermograms of TGAP/DDS.

and the cure peak temperatures shift to higher temperatures with decreasing curing agent concentration. Table I shows the peak exotherm temperature $\left(T_{\mathrm{p}}\right)$ and the extents of cure at the peak $\left(\alpha_{p}\right)$ as a function of heating rate. It has been observed that heat of cure $\left(H_{\mathrm{c}}\right)$ increases with decreasing the curing agent concentration. At a constant curing agent, the extents of cure at the peak is independent of heating rate. It has been also observed that peak exotherm temperature increases with decreasing DDS concentration at a given heating rate. From the table, it can be concluded that the extents of cure at the peaks are nearly constant and independent of heating rate at a given DDS concentration.

Ozawa proposed a simple relationship between activation energy $\left(E_{\mathrm{a}}\right)$, heating rate $(\Phi)$, and peak exotherm temperature $\left(T_{\mathrm{p}}\right)$ under the assumption that the extent of reaction at the peak exotherm is constant and independent of heating rate. ${ }^{11,12}$

$$
\Delta\left(1 / T_{\mathrm{p}}\right) / \Delta(\ln \Phi)=-R /\left(1.052 E_{\mathrm{a}}\right)
$$

By using Dolye's approximation, Ozawa 
Table I. Peak temperature $\left(T_{\mathrm{p}}\right)$ and the conversion at the peak $\left(\alpha_{\mathrm{p}}\right)$ from dynamic scan for $\mathrm{HDl}-3$

\begin{tabular}{|c|c|c|c|c|c|c|c|c|c|}
\hline \multirow{3}{*}{$\begin{array}{l}\text { Heating } \\
\text { rate } \\
{ }^{\circ} \mathrm{C} \mathrm{min}^{-1}\end{array}$} & \multicolumn{3}{|c|}{ HD1 } & \multicolumn{3}{|c|}{ HD2 } & \multicolumn{3}{|c|}{ HD3 } \\
\hline & $T_{p}$ & & $E_{\mathrm{a}}$ & $T_{\mathrm{p}}$ & & $E_{\mathrm{a}}$ & $T_{\mathrm{p}}$ & & $E_{\mathrm{a}}$ \\
\hline & ${ }^{\circ} \mathrm{C}$ & $p_{p}$ & $\mathrm{~kJ} \mathrm{~mol}^{-1}$ & ${ }^{\circ} \mathrm{C}$ & $\mathrm{p}$ & $\mathrm{kJ} \mathrm{mol}^{-1}$ & ${ }^{\circ} \mathrm{C}$ & $p_{p}$ & $\mathrm{~kJ} \mathrm{~mol}^{-1}$ \\
\hline 2.5 & 215 & 0.67 & & 244 & 0.83 & & 253 & 0.86 & \\
\hline 5.0 & 237 & 0.61 & 69.7 & 260 & 0.84 & 94.5 & 268 & 0.86 & 99.1 \\
\hline 10 & 261 & 0.64 & & 277 & 0.83 & & 285 & 0.85 & \\
\hline 20 & 282 & 0.65 & & 295 & 0.84 & & 304 & 0.86 & \\
\hline
\end{tabular}

derived the following approximate relationsip. ${ }^{19}$

$$
\log \Phi=C-0.4567 E_{\mathrm{a}} / R T_{\mathrm{p}}
$$

The activation energy can be obtained from peak exotherm temperature as a function of heating rate. Derived approximation equation is fairly accurate for $30 \leq E_{\mathrm{a}} / R T \leq 50$. Duswalt reported that the activation energy can be obtained with precision of $\pm 3 \%$ and estimated accuracy of $\pm 10 \% .^{13}$

Figure 3 represents the reciprocal peak temperature as a function of logarithm heating rate. From the figure, there are good linearity and similar slope with different DDS concentration. This phenomenon has been expected on the basis that the activation energy for cure reaction should be only dependent on the kind of the curing agent.

\section{Isothermal Cure Kinetics}

Figure 4 illustrates isothermal DSC thermograms for HD1. It has been observed that cure rate passes through a maximum point and the cure rate at peak $\left(\alpha_{\mathrm{p}}\right)$ increases with increasing in cure temperature. The other two formulations show analogous trends. These facts indicate that cure reaction of TGAP/DDS system follows the autocatalytic reaction.

Plots of degree of conversion against cure time for HD1 are shown in Figure 5. At a given time, the degree of conversion increases with increasing cure temperature. The time conver-

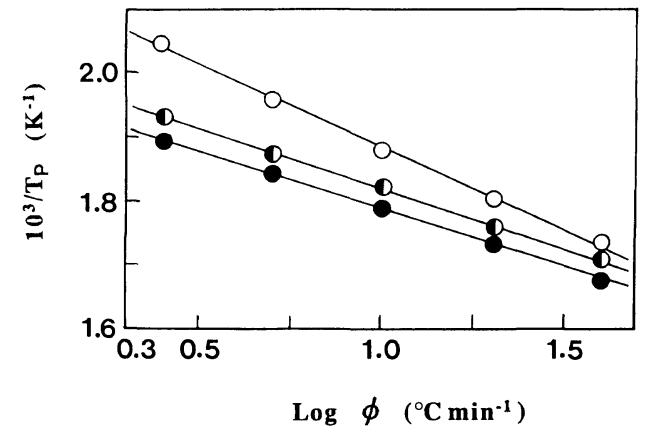

Figure 3. Reciprocal peak temprature as a function of logarithm heating rate for HD1 (O), HD2 (O), and HD3 (O).

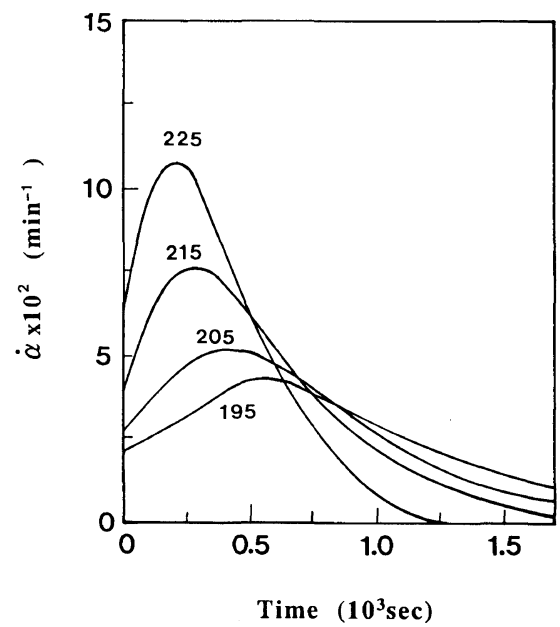

Figure 4. Cure rate as a function of cure time for HD1.

sion curves for HD2 and HD3 show the same trend, but shift along the time axis according to the cure time. 


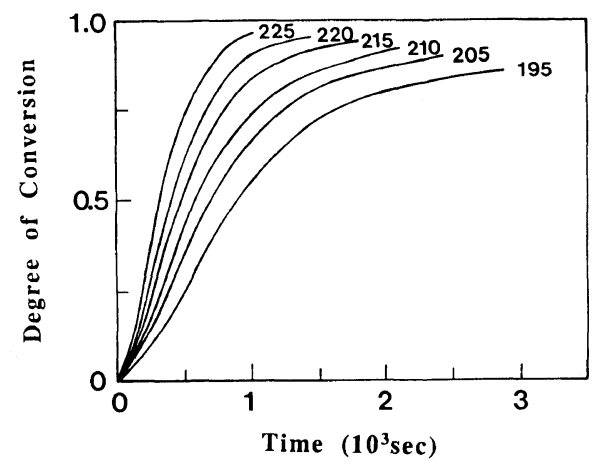

Figure 5. Degree of conversion versus cure time for HD1.

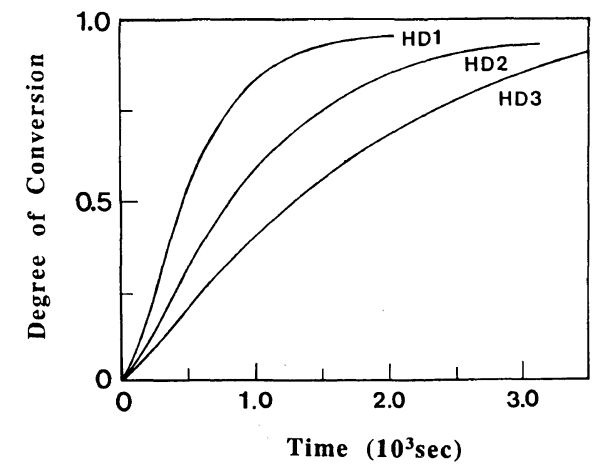

Figure 6. Degree of conversion with different epoxy formulation.

Figure 6 shows the degree of conversion as a function of cure time. The degree of conversion increases with increasing DDS concentration at a given time. It can be explained that the reaction contribution of epoxide/primary amine may be relatively decreased for overall epoxy cure reaction due to the decrease of DDS concentration.

The cure rate as a function of DDS concentration is illustrated in Figure 7. As the DDS concentration increases gradually, the cure rate increases drastically. In the case of HD3, second exotherm peak has been observed in reaction rate curve. At a constant cure temperature, cure rate for HD3 was plotted as a function of the degree of cure in Figure 8. In this figure, second exotherm peak begins to appear at approximately $50 \%$ conversion. Morgan et al. reported that primary amine-

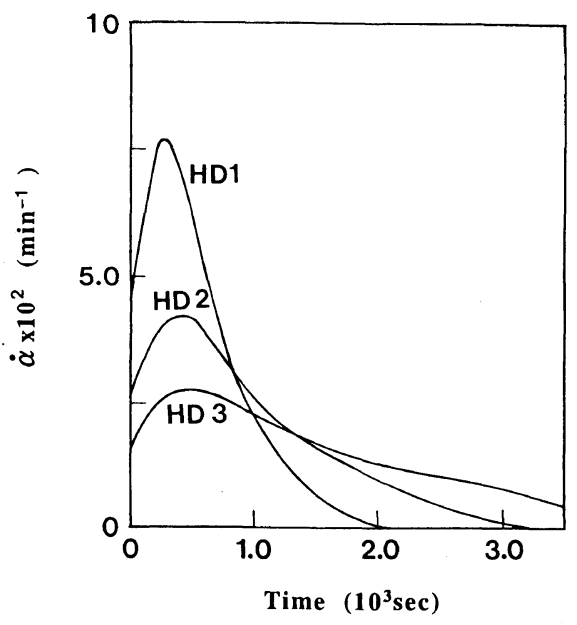

Figure 7. Cure rate as a function of cure time.

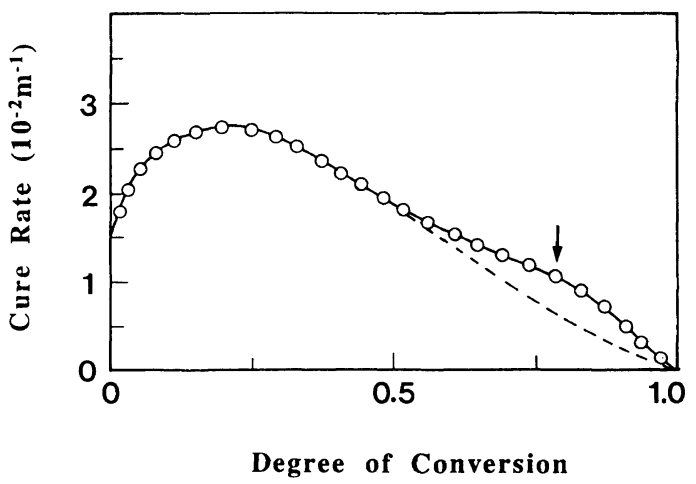

Figure 8. Cure rate as a function of degree of conversion for HD3.

eopoxide reaction dominantes at the early stage of cure and epoxide-hydroxyl reaction dominates at the later stage of cure. ${ }^{12}$ Mijovic et al. studied the cure kinetics of TGDDM/DDS system using DSC. ${ }^{11}$ They observed second exotherm peak at the low concentration of DDS during isothermal cure. In this study, TGAP/DDS system has second exotherm peak at the high concentration of DDS compared to TGDDM/DDS. This may be due to structural difference of TGAP resin. Cure process of epoxy is mainly dependent on the structure and functional group of epoxy resin.

The kinetic rate constant $k_{1}$, which was the initial reaction rate at a given temperature, was 


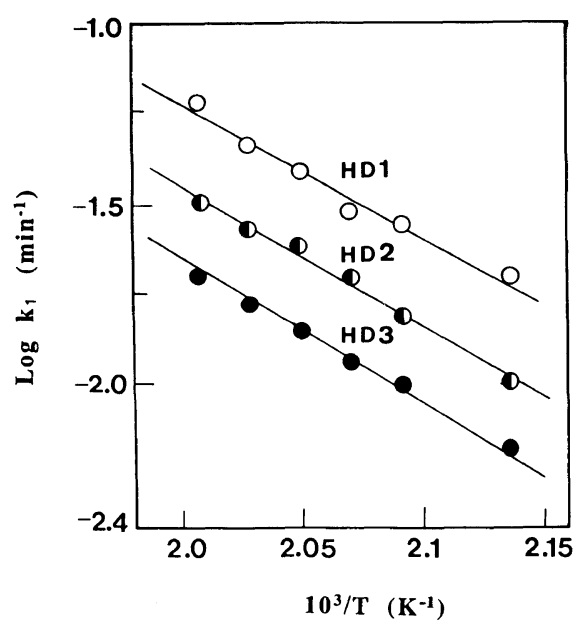

Figure 9. Initial reaction rate constant $\left(k_{1}\right)$ versus reciprocal peak temperature.

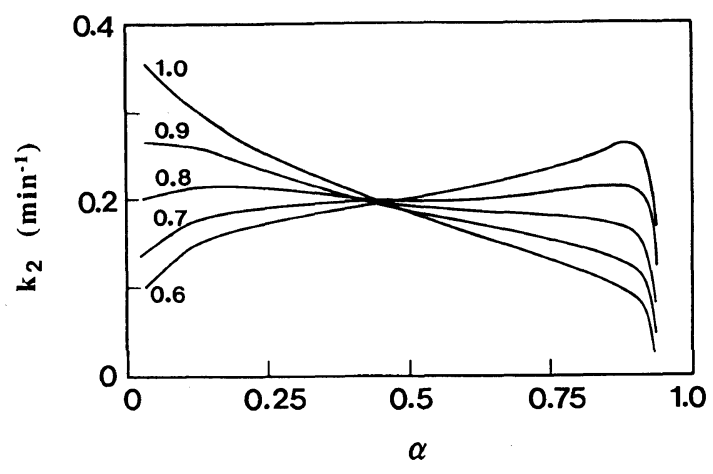

Figure 10. Cure rate constant $\left(k_{2}\right)$ as a function of degree of Conversion $(\alpha)$ with various $m$ values for HD1.

determined directly from the reaction rate curve. The value of $k_{1}$ was found to follow the Arrhenius dependence on cure temperature in Figure 9. From the figure, it has been observed that the activation energy for epoxy reaction increases with decreasing DDS concentration. As we mentioned previously, primary amineepoxide reaction dominates at the high concentration of DDS and this reaction has the lower activation energy compared to the hydroxyl-epoxy reaction. It can be explained that the activation energy of epoxy reaction has been reduced by this process at the high DDS concentration.

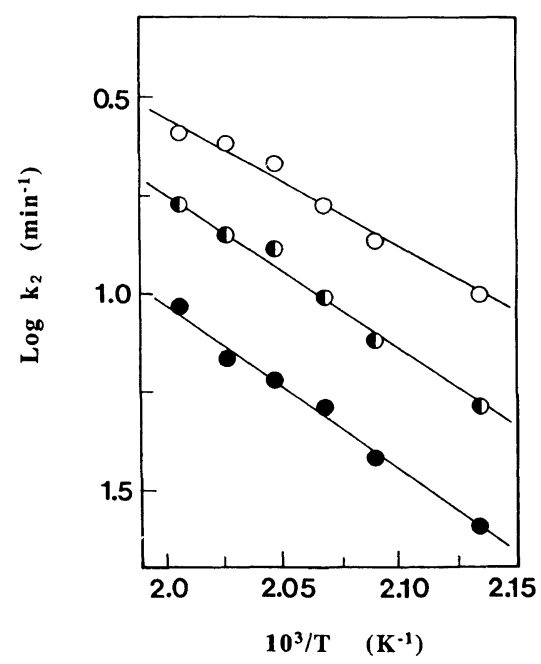

Figure 11. Logarithm reaction rate constant $\left(k_{2}\right)$ as a function of cure temperature for fomulation HDl (O), HD2 (๑), HD3 (๑).

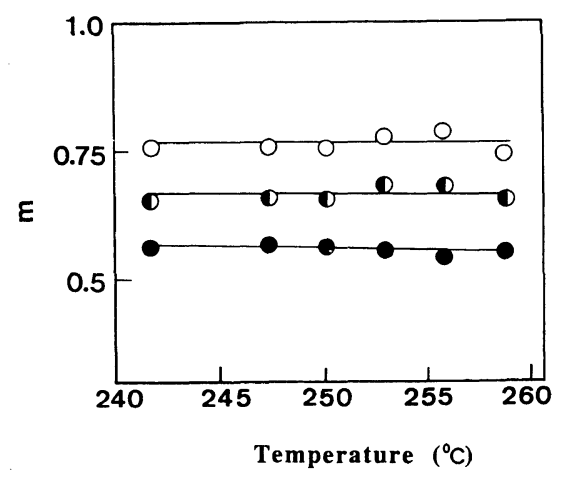

Figure 12. Kinetic Parameter $m$ as a function of cure temperature.

Substituting arbitrary values of $m$, kinetic rate constant $\left(k_{2}\right)$ and kinetic exponent $(m)$ can be directly determined from eq 1 under the assumption of $m+n=2 .{ }^{17}$ When $k_{2}$ value against the degree of conversion is constant, $m$ and $k_{2}$ value would be determined automatically. The kinetic rate constants $\left(k_{2}\right)$ as a function of conversion with $m$ values as a parameter at $215^{\circ} \mathrm{C}$ for $\mathrm{HD} 1$ are shown in Figure 10. It has been observed that the $k_{2}$ values are nearly constant in the range of $m$ values which are $0.85-0.90$ for HD1. The 
DSC Cure Studies of High Performance Epoxy Resins. I

Table II. Summary of kinetic parameters for HD1-3

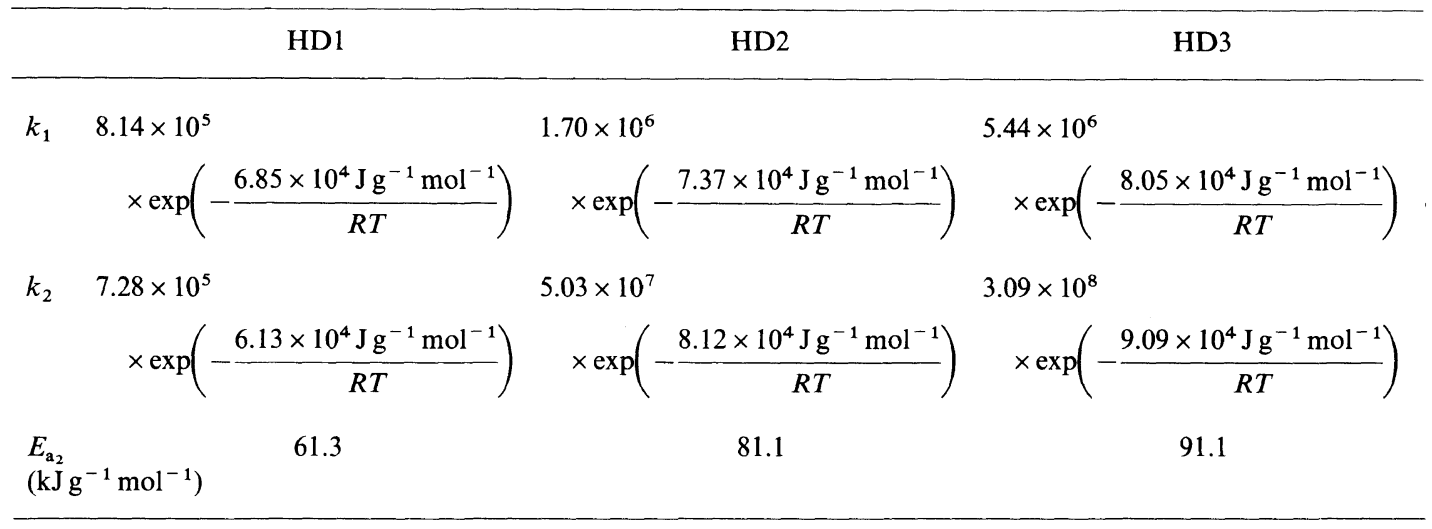

reaction rate constant $\left(k_{2}\right)$ as a function of reciprocal cure temperature is plotted in Figure 11. The temperature dependence of the reaction rate constant fits well the Arrhenius form. From the figure, it has been observed that the activation energy for the cure reaction of epoxy resin decreases with increasing DDS concentration. As we mentioned previously, primary amine-epoxide reaction dominantes at the high concentration of DDS. This reaction has the lower activation nergy compared to the hydroxyl-epoxide reaction. It can be explained that the activation energy for the cure reaction of epoxy resin has been reduced by this process at the high DDS concentration.

Figure 12 shows the kinetic parameter $m$ value as a function of cure temperature. It was observed that $m$ value was independent of cure temperature as a given formulation. However the value increased with increasing DDS concentration.

A summary of the corresponding kinetic parameters obtained from the isothermal experiment for HD1-3 is shown in Table II. Prime reported that the dynamic kinetic parameters measured on epoxies were larger than the isothermal. ${ }^{20}$ Compared with the dynamic data in Table I, the activation energy from isothermal experiment had good agreement with Prime's result. The activation energy of TGAP/DDS system was slightly higher than that of TGDDM/DDS system reported in the literature. ${ }^{18,22}$

\section{REFERENCES}

1. R. S. Bauer, "Epoxy Resin Chemistry I," The American Chemical Society, Washington, D.C., 1979.

2. R. S. Bauer, "Epoxy Resin Chemistry II," The American Chemical Society, Washington, D.C., 1983.

3. C. A. May and O. Tanaka, "Epoxy Resins Chemistry and Technology," Marcel Dekker, Inc., New York and Basel, 1983.

4. G. Lubin, "Handbook of Composites," 57-58, Van Nostrand Reinhold, New York, 1984.

5. C. A. May, "Epoxy Resin Chemistry and Technology," Marcel Dekker, New York and Basel, 1988.

6. R. S. Bauer, A. G. Filippov, L. M. Schlaudt, and W. V. Breitigam, The Processings of 32nd International SAMPE Symposium, April 6-9, 1987, pp 1104 1113.

7. R. S. Bauer, A. G. Filippov, L. M. Schlaudt, and W. V. Breitigam, The Processings of 33rd International SAMPE Symposium, March 7-10, 1988, pp 1385-1393.

8. R. S. Bauer, The Proceedings of 34th International SAMPE Symposium, May 7-11, 1989, pp 18891990.

9. L. M. Schlaudt, R. S. Bauer and C. A. Blackburn, The Proceedings of 34th International SAMPE Symposium, May 8-11, 1989, pp 917-928.

10. L. Schechter, J. Wynstra, and R. P. Kurkjy, Ind. Eng. Chem., 48, 94 (1956).

11. T. Ozawa, Bull. Chem. Soc. Jpn., 38, 1881 (1965).

12. T. Ozawa, J. Therm. Anal., 2, 301 (1970).

13. A. A. Duswalt, Thermochim. Acta., 8, 57 (1974).

14. M. R. Kamal, S. Sourour, and M. Ryan, Soc., Plast. Eng. Tech. Papers, 19, 187 (1973). 
15. S. Sorour and M. R. Kamal, Therms Chem. Acta., 14, 41 (1976).

16. A. Dutta, MSc Thesis, State University of N.Y. at Buffalo, Amherst, N.Y. (1970).

17. M. E. Ryan and A. Dutta, Polymer, 20, 203 (1979).

18. J. Mijovic, J. Kim, and J. Slaby, J. Appl. Polym. Sci., 29, 1449 (1984).

19. K. J. Morgan, J. A. Happe, and E. T. Mores, 28th
National SAMPE Symposium Anaheim, Calif. April 12-14 (1983).

20. C. D. Doyle, Anal. Chem., 33, 77 (1961).

21. R. B. Prime, Polym. Eng. Sci., 13(5), 365-371 (1973).

22. R. B. Prime, "Thermal Characterization of Polymeric Materials," E. A. Turi, Ed., Academic N.Y. 435-569 (1981). 